\title{
Analysis of Components and Circuit for FMCW Radar System
}

\author{
${ }^{1}$ SAQIB AHMED, ${ }^{1}$ FAHIM AZIZ UMRANI, AND ${ }^{1}$ ABDUL BASSIT SURAHIO \\ ${ }^{1}$ Institute of Information \& Communication Technologies, Mehran University of \\ Engineering \& Technology, Jamshoro, Pakistan. \\ saqibahmedmemon34@gmail.com, faheemaziz.umrani@faculty.muet.edu.pk, \\ absurahio@gmail.com
}

\section{Abstract}

In this paper, the components required for FMCW (Frequency-Modulated Continuous Wave) RADAR (Radio Detection and Ranging) system operating at $3 \mathrm{GHz}$ i.e. modulator circuit and video amplifier circuit are designed and tested. For this purpose, the RF (Radio Frequency) components were selected from microstrip trainer kit MST-523 of Feedback Instruments. The working of each component is analyzed through VNA (Vector Network Analyzer) and spectrum analyzer. The patch antennas provided in the kit were used as transmitter and receiver operating at $3 \mathrm{GHz}$. The modulator circuit is built with variable frequency and amplitude to modulate the signal from VCO (Voltage Controlled Oscillator). The triangular wave is selected for this purpose to modulate the VCO. The received signal is measured on spectrum analyzer and received power is measured at every integral multiple of wavelength to check working state of antenna and change in power. The Low pass filter circuit is also designed to filter out the higher frequencies after the mixer stage. The RADAR range is calculated based on measurement of the system.

Key Words: $\quad$ Radar, Frequency Modulated Continuous Wave, Patch Antenna.

\section{INTRODUCTION}

There has been much progress in various wireless systems and networks since the 19 th century. The radio signals ubiquitously are propagating all around us. RADAR system is one example of a wireless system which has been developed since 1935 when Sir Robert Watson-Watt reported the first practical RADAR system. RADAR systems are used for surveillance, human tracking, remote sensing, positioning and location-based applications [1]. The RADAR is an extension of the human sensing technology that offer new potentials. It detects and tracks target that are at far distances that cannot be identified by naked eyes. It can work irrespective of having mist, smoke and clouds. Among the popular microwave radars FMCW radar is vital and used in diverse applications [2]. 
Based on different types of waveforms, RADAR systems can be classified into CW (Continuous-Wave) RADAR and pulsed radar. Pulsed radar blind spot in front of radar of $50 \mathrm{~m}$ or more leading to poorer resolution. On the other hand, $\mathrm{CW}$ radar offers less complex architecture making it easy to integrate into system also consumes less power. All these characteristics of $\mathrm{CW}$ radar makes it better candidate for movable and portable applications.

Different sensors are utilized for human tracking, the most obvious method is usage of camera along with an image processing and feature recognition algorithm. All these camera-based approaches often lead to high cost implementations and do not provide security. Moreover, they also require LOS (Line of Sight) approach. Other detection concepts use acoustic waves or non-visible light which can provide more privacy [3].

Among these approaches, RADAR-based system due to their electromagnetic waves have got benefits as compared since it can penetrate through walls. In comparison to LiDAR (Light Detection and Ranging) and infrared sensors, radar systems also perform well in rough environment and are less prone to mist, dust or rain. Furthermore, FMCW radar have great short-range results because of strong environmental robustness, good sensitivity and no blind spots [4].

\section{FMCW RADAR}

From its name it suggests that, FMCW radar is a technique to get distance information by modulating frequency of CW signal. This technique has been used in past and has long history but was restricted to specific applications such as radio altimeters. Though, this technique has gained more interest because of following reasons listed in [5]. The first benefit is that modulation is easily compatible with wide variety of solid-state transmitters. The second benefit is that the measurement of frequency which is required for extracting range information from radar can be performed digitally, for example, MATLAB integration with system will have digital processing of signal thereby extracting range. Another example is the integration of Arduino/FPGA (Field Programmable Gate Array) standalone microcontroller can make the system portable as well as digital processor of signal.

Following are the advantages of FMCW radar compared to other types of modulated CW.

(i) The ability to control the range and to obtain very efficient use of spectrum.

(ii) The range resolution can be changed with ease and high range resolution can also be obtained without requiring video bandwidth and IF (Intermediate Frequency). 
Before the digital processing era many earlier FMCW radars utilized single band-pass to receive signal at a single range and sweep rate was changed periodically to look all the possible range [6]. Which was making it cumbersome in radar tracking. After the use of digital processing, analyzation was easy for mixture of beat frequencies of multiple targets at different ranges.

Charvat et. al. [7], the MIT (Massachusetts Institute of Technology) university gave the course on design of radar using Coffee cans as antennas and system was designed by connecting individual radio frequency components by SMA (Sub-Miniature Version-A) connectors. The system was based on FMCW wave signal operating at $2.4 \mathrm{GHz}$ frequency with about $10 \mathrm{~mW}$ power for transmit.

In recent advancement, FMCW radar has been used in many applications from military area to civil applications. FMCW radar can be used in automotive area for recording velocity violation on traffics. It can be used as intelligence driving systems to avoid collision. FMCW applications are also employed for marine navigation and ship identification. Some researchers employed the use of FMCW radar in geosciences field for wind speed and direction measurement of wind [8]. On the other side FMCW has great application in medical and health areas such as fall detection of elderly people and breath detection.

In this work the, the received power of patch antennas operating at $3 \mathrm{GHz}$ is analyzed for FMCW RADAR system. The system is designed using RF components of the training kit MST-523 by the make of Feedback instrument including VCO operating at frequency from $2.4-3.7 \mathrm{GHz}$.

\section{METHODLOGY}

Pulsed radar work by emitting a short pulse to measure the distance of target and observe the time of flight of returned signal. This system requires to have high transmit power and mostly physical apparatus are expensive. In comparison to this the FMCW radar do the same with much smaller transmit power and having smaller physical apparatus. The FMCW RADAR's block diagram is shown in Fig.1. 


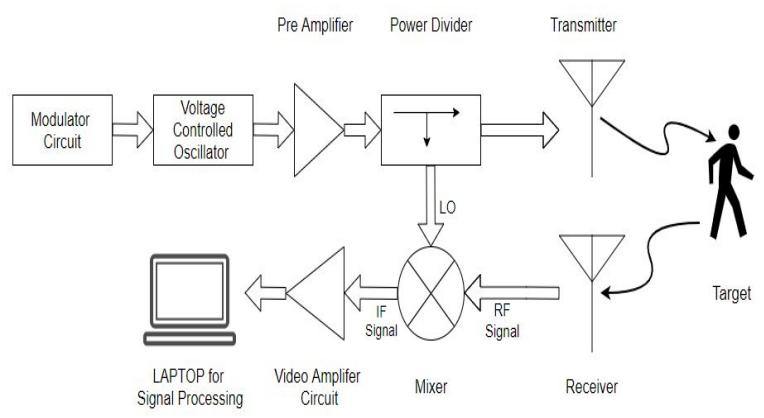

FIG. 1. FMCW RADAR BLOCK DIAGRAM

The FMCW RADAR emits the continuous microwave signal which is frequency modulated having the duration greater then return signal such as triangular function of period $\mathrm{T}$ illustrated in Fig. 2. To understand more the return signal received after hitting the target is replica of transmitted signal with some frequency shift and delay [9] as shown in Fig. 2. Then, at the receiver the echo is mixed with the transmitted signal. This type of system is known as homodyne system because the transmitted signal is used for down conversion. After low pass filtering the differential frequency is obtain which is called IF frequency which is approximately sinusoidal fw which is constant in time interval T- $\tau$ as shown in Equation (1), also illustrated in Fig.2.

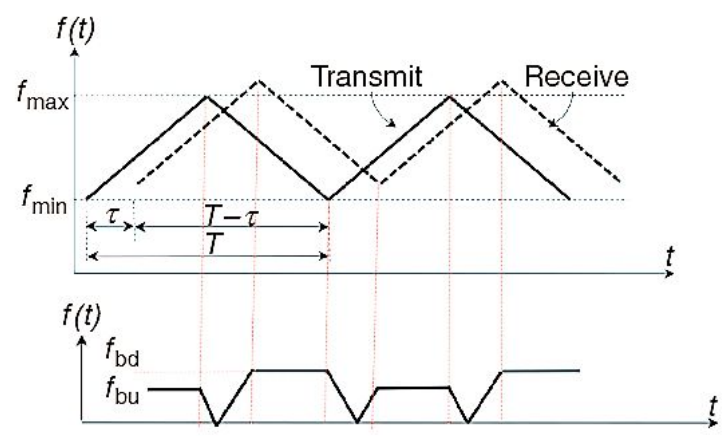

FIG. 2. THE ILLUSTRATION OF TRANSMITTED, RECEIVED AND BEAT SIGNAL AS A FUNCTION OF TIME

$\mathrm{fw}=\alpha \tau$

where $\alpha=\Delta \mathrm{f} / \mathrm{T}$ represents modulation waveform slope and $\Delta_{\mathrm{f}}=\mathrm{f}_{\max }-\mathrm{f}_{\min }$ is the maximum frequency deviation. The doppler shift is obtained from the received signal of the moving target, the change in signal between doppler shifted signal and transmitted signal is called beat signal. The beat frequency will be used for down chirp and up chirp $\mathrm{f}_{\mathrm{bu}}$ and $\mathrm{f}_{\mathrm{bd}}$. The range beat frequency $\mathrm{f}_{\mathrm{r}}$ and the Doppler frequency $f_{d}$ are mathematically expressed as in Equations (2-3): 
$f_{r}=\frac{\left|f_{b u}+f_{b d}\right|}{2}$

$f_{d}=\frac{\left|f_{b u}+f_{b d}\right|}{2}$

By the help of signal processing of the video signal in software such as MATLAB, $f_{r}$ and $f_{d}$ can be obtained. Also, the target velocity and distance can be calculated by Equations (4-5), as:

$$
\begin{aligned}
& R=\frac{c T f_{r}}{2 B} \\
& V=\frac{c f_{d}}{2 f_{c}}
\end{aligned}
$$

where $\mathrm{R}$ is range, $\mathrm{V}$ is velocity, and $\mathrm{B}$ is the bandwidth of the modulation frequency [10].

\section{IMPLEMENTATION}

The implementation of this system is in such a way that in first phase the RF components are tested on spectrum analyzer/VNA. After checking of RF components, the second phase is of circuit designing that includes modulator circuit for VCO and amplifier circuit for IF signal from mixer. They are made and verified on oscilloscope. In third phase these circuits are combined with RF components and RADAR system is designed. Testing and Measurement of the system are performed. The illustration of phases is shown in Fig. 3. This implementation section is discussed in such a way that each circuit is briefly explained in separate sections. Also, the RF circuit is explained briefly with measurements separately. 


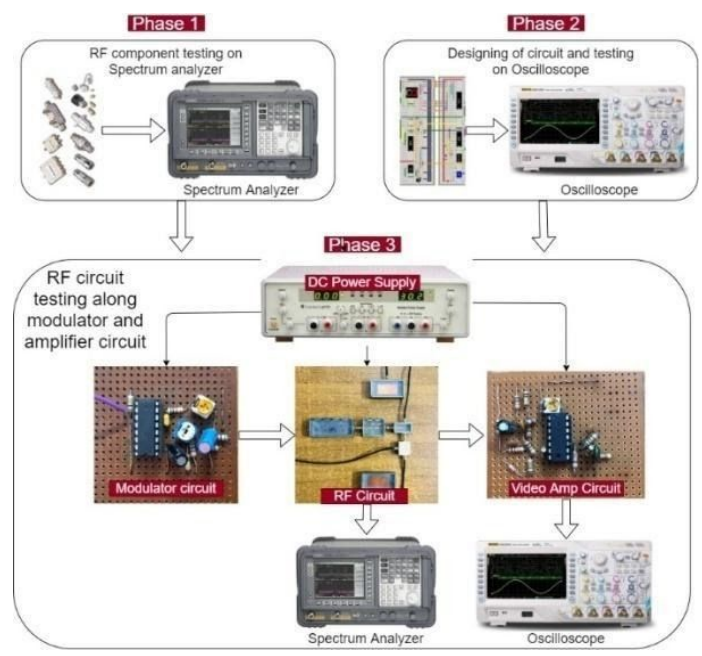

FIG. 3. ILLUSTRATION OF IMPLEMENTATION OF PHASES

\section{Modulator Circuit}

In this study we have used a FMCW RADAR. Basically, there are various waveforms that can be used for FM chirping. We have chosen triangular wave chirp and analyzed the circuit at component level.

Fig. 4 represent how we produce our FMCW signal. Other options for modulating signal are square, sawtooth, sine waves. Only a triangle modulation will give both range and velocity.

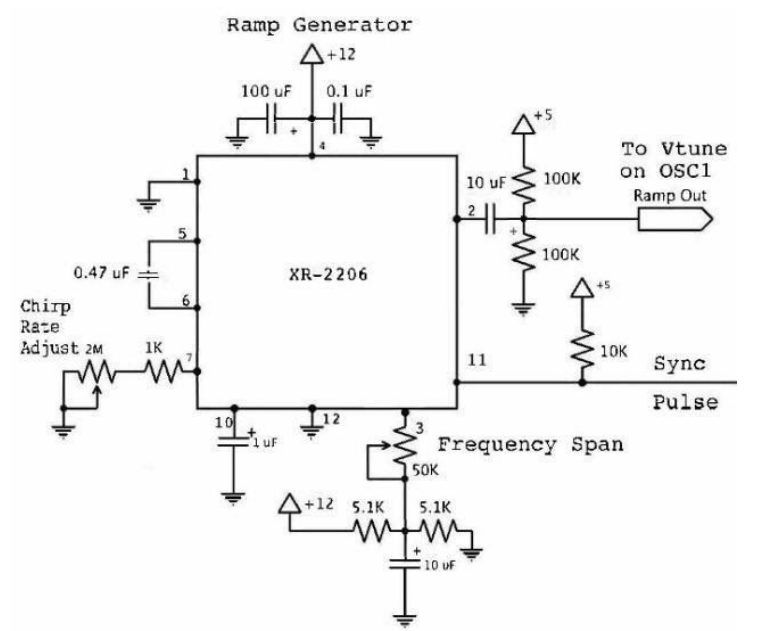

FIG. 4. XR-2206 CHIRP WITH FMCW TRIANGLE WAVE IMPLEMENTATION

Fig. 5 is schematic circuit for sine wave generation given by the IC manufacturer datasheet. Referring to the above XR-2206 datasheet, the pin 13 and pin 14 that is S1 should be open for triangular wave and closed for sine wave. Output is taken from pin 
2 and for synchronization purpose square wave output is taken from Pin 11. Hardware implementation of modulator circuit is shown in Fig. 6.

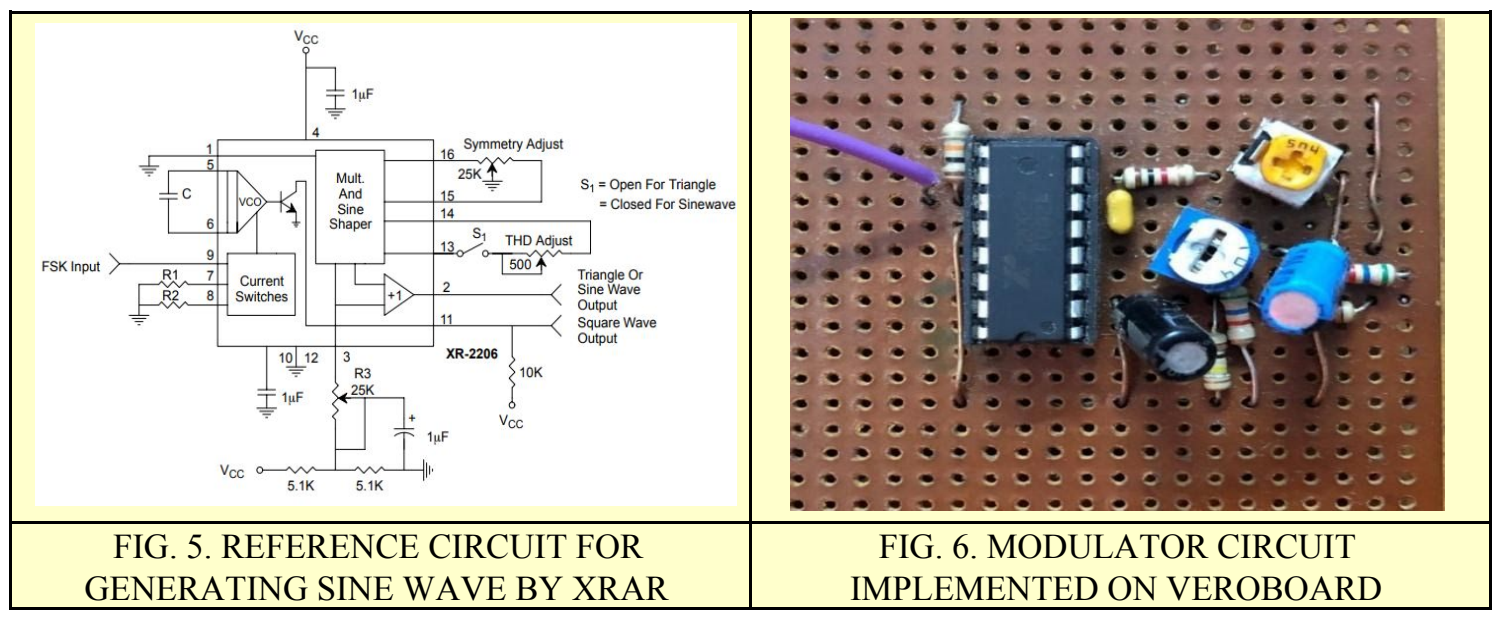

Next, we select the values of $\mathrm{R}$ and $\mathrm{C}$ for Pin 5, 6 and 7 for chirp rate adjust and frequency span. Choosing $\mathrm{C}=0.47 \mathrm{uF}$ and value of $\mathrm{R}$ through potentiometer to get frequency nearly $40 \mathrm{~Hz}$. The span of frequency is proportional to the potentiometer R3. After setting "Span" and "Chirp Frequency" pins set up, we should ground the pins like pin 1 which is for to modulate output amplitude that is not required. Then we apply voltage of $12 \mathrm{~V}$ to Vcc (Voltage at the common collector) pin with two parallel capacitors to control ramp.

Fig. 7 shows the output at pin 2, which is connected to oscilloscope also the sync pulse is connected to other probe of oscilloscope for synchronization purpose. Where the frequency is $40 \mathrm{~Hz}$ which can be varied from the potentiometer. Ensuring the proper voltage at each pin in necessary if not then clipping will occur.

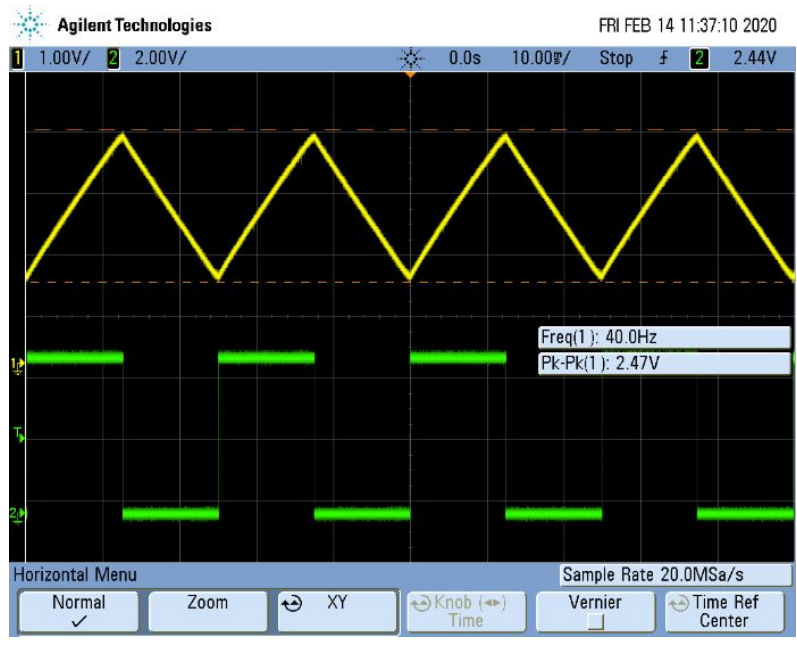


FIG. 7. THE OUTPUT OF MODULATOR CIRCUIT CONNECTED TO OSCILLOSCOPE

\section{Video Amplifier Circuit}

The signal received by receiver antenna is fed to the mixer RF port and mixer's LO port is fed by out of power divider. Mixer compares two signals and IF is created. That IF signal is fed to the amplifier circuit. The amplifier circuit is also used to make active low pass filter and is made by single supply configuration. In the gain stage the IF signal is amplified. The signal is set to maximum value before the circuit clips it during FMCW mode. Then the signal is followed by $15 \mathrm{KHz} 4$ th order low pass filter to remove the higher frequencies components, which were created from mixer stage.

Fig. 8 shows the schematic layout for video amplifier circuit. The quad op-amp IC LM324 is used [11]. The $15 \mathrm{KHz}$ low-pass filter circuit was tested separately using the signal generator as input. The Fig. 9 show the output of low pass filter section of video amplifier. The input signal of $15 \mathrm{KHz}$ was adjusted to $10 \mathrm{Vp}-\mathrm{p}$ and measured the output signal on the oscilloscope is $4.25 \mathrm{Vp}-\mathrm{p}$. Calculating the attenuation from input and output signal values, the attenuation comes out to be $7 \mathrm{~dB}$ for $15 \mathrm{KHz}$. The Fig. 10 is illustration of hardware implemented on Veroboard.

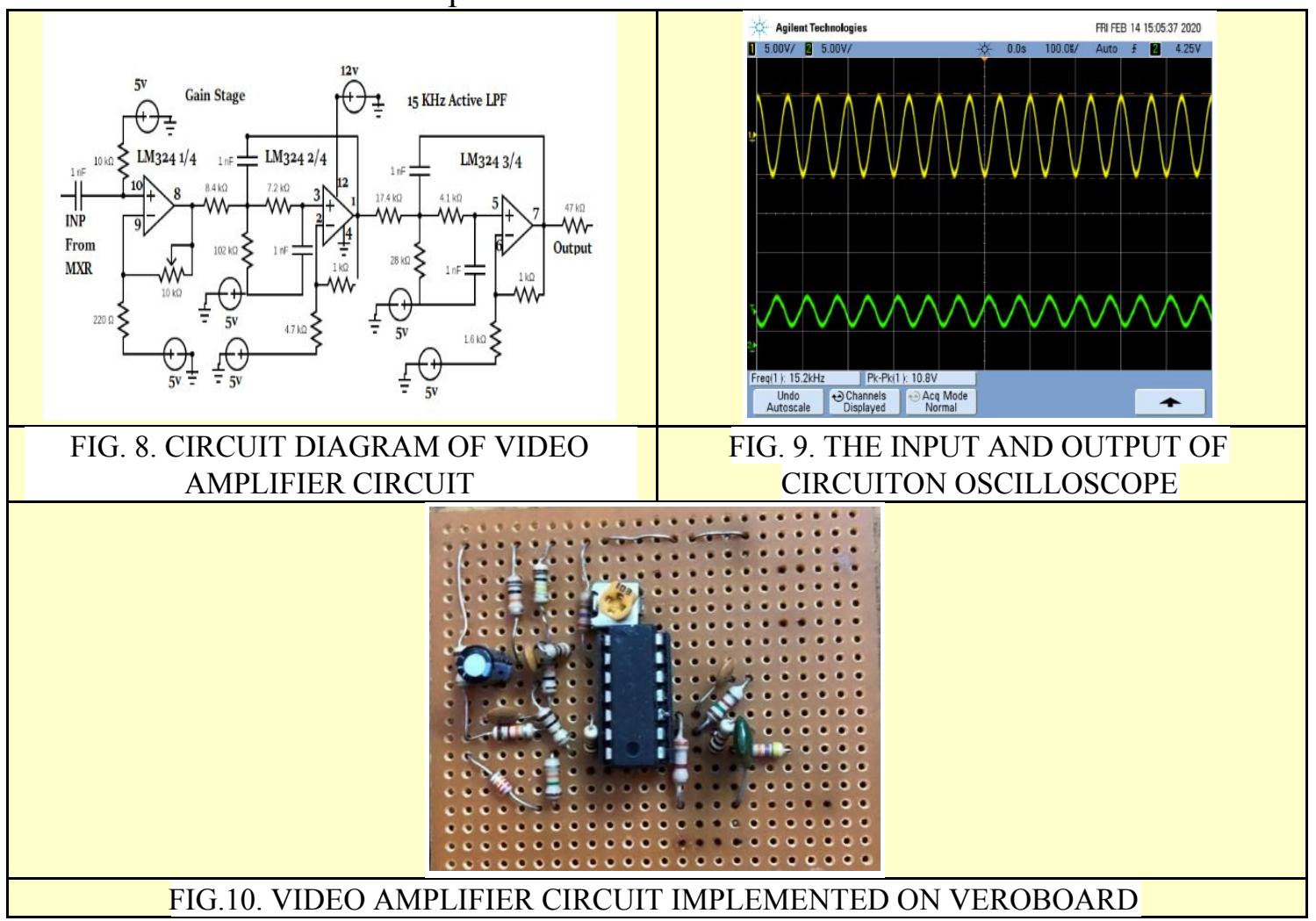

RF Circuit 
The first component of FMCW RADAR is VCO which is producing signal with power of $12.2 \mathrm{dBm}$ and enters into the power amplifier. The power amplifier amplifies the signal up to $19.5 \mathrm{dBm}$. This amplified signal enters into power divider. The power divider has two outputs ports and divide the signal at the input port into two identical signals of power $15.5 \mathrm{dBm}$. The first signal goes into transmitting antenna and the output from second port goes to the mixer LO (Local Oscillator) port. There is power loss of $-4 \mathrm{dBm}$ in power divider stage. The layout of developed RF circuit is illustrated in Fig. 11.

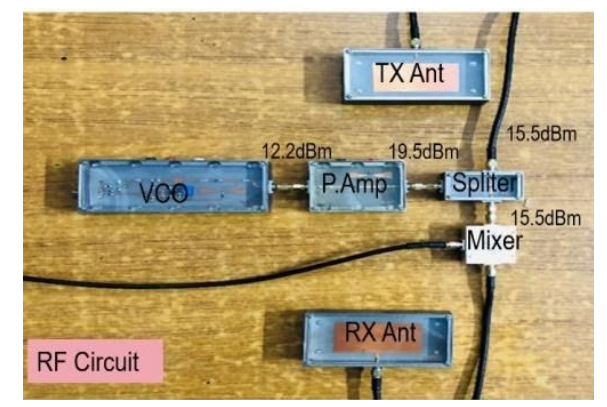

FIG. 11. RF CIRCUIT FOR FMCW RADAR

The receiving antenna receive the reflected RF signal and fed it to the RF port of mixer. The mixer brings down the RF signal to an IF signal. The RF signal is followed by video amplifier circuit where the signal is cleared from higher frequencies.

The Patch antenna which is used has operating frequency of $3 \pm 0.05 \mathrm{GHz}$. Therefore, the wavelength for this frequency is $10 \mathrm{~cm}$ thus its half wavelength will be $5 \mathrm{~cm}$. The received power was observed with spectrum analyzer Agilent-E4407B. The VCO was operated with $15 \mathrm{~V}$ de supply and was tuned to operate at $3 \mathrm{GHz}$ with tuning input port. The signal was received by receiving antenna which was fed to spectrum analyzer.

It is noted that the received power was maximum at every integral multiple of wavelength and minimum at the half of the wavelength of each integral multiple as illustrated in Fig.12. 


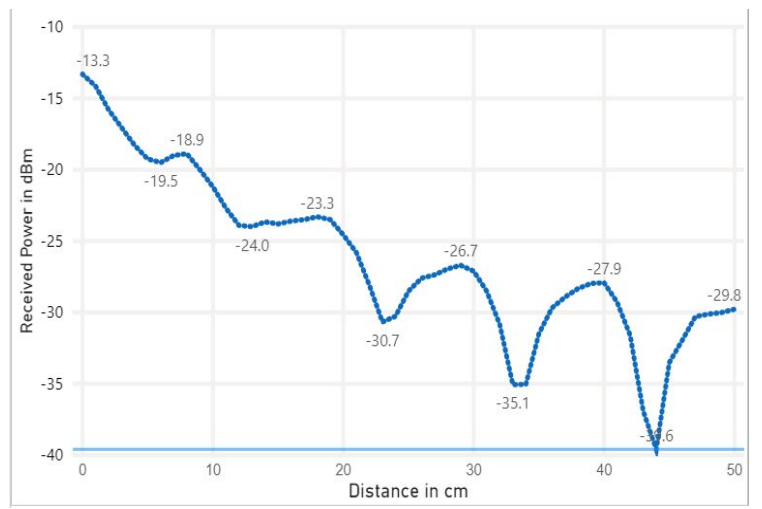

FIG. 12. GRAPH PLOTTED FOR RECEIVED POWER AGAINST DISTANCE

Based on the measurement of received power and fixed value antenna gain, frequency of antenna and radar cross section, the range of radar is calculated by formula in Equation (6). The range coming out to be $20 \mathrm{~m}$ for patch antenna.

$$
\text { Range }=4 \sqrt{\frac{\mathrm{P}_{\mathrm{r}} \times \sigma \times \mathrm{c}^{2} \times \mathrm{G}_{\mathrm{r}}^{2}}{(4 \pi)^{3} \times \mathrm{f}_{0}^{2} \times \mathrm{P}_{\text {min }}}}
$$

\section{CONCLUSION}

The FMCW radar system is designed. Each components of system are tested using spectrum analyzer. $\mathrm{VCO}$ was operated from $2.4-3.7 \mathrm{GHz}$ to check which frequency is best for patch antenna. Then $3.0 \mathrm{GHz}$ was selected as center frequency for patch antenna.

The circuit for modulator and amplifier circuit used in the system are tested and verified on breadboard first then soldered on Veroboard to avoid any losing of wire during the testing and measurement phase.

With this research work, in-depth knowledge was gained about modulator circuits, RF system components, it's testing and measurement. In future work, the full system design will be made with all these component level research and measurement. The FMCW radar can be tested with different types of antenna to have more directivity, improved performance and range. It is also possible to implement the system with advanced analogue to digital convertor.

\section{ACKNOWLEDGEMENT}




\section{Gyancity Journal of Electronics and Computer Science, \\ Vol.5, No.1, pp. 1-11, March 2020 \\ ISSN:2446-2918DOI: 10.21058/gjecs.2020.51001}

Authors very thankful to Department of Telecommunication Engineering, Mehran University of Engineering \& Technology, Jamshoro, Pakistan, for providing the environment to carry out the research work successfully.

\section{REFERENCES}

[1] Umrani, F.A., Chowdhry, B.S., and Umrani, A.W., "Simulation Design of Doppler Filter Bank (DFB) for Pulsed DopplerRadar to Measure Wind Velocities", Mehran University Research Journal of Engineering \& Technology, Volume 29, No. 1, pp. 91-98, Jamshoro, Pakistan, January, 2010.

[2] El-Mokdad, S., Khrayzat, M., and Bazzi, A., "FMCW Implementation on LabVIEW", International Conference on Computer and Application, 2018.

[3] Hussein, M., Abd-Almageed, W., Ran, Y., and Davis, L., "Real-Time Human Detection, Tracking, and Verification in Uncontrolled Camera Motion Environments", IEEE $4^{\text {th }}$ International Conference on Computer Vision Systems, pp. 41-41, January, 2006.

[4] Ralston, T.S., Charvat, G.L., and Peabody, J.E., "Real-Time Through-Wall Imaging Using an Ultrawideband Multiple-Input Multiple-Output (MIMO) Phased Array Radar System", IEEE International Symposium on Phased Array Systems and Technology, pp. 551-558, October, 2010.

[5] Stove, A.G., "Linear FMCW Radar Techniques", IEEE Proceedings-F, Volume 139, No. 5, pp. 825-830, 1992.

[6] Pyo, G., Kim, C.-Y., and Hong, S., "Single Antenna FMCW Radar CMOS Transceiver IC", IEEE Transactions on Microwave Theory and Techniques, Volume 2, pp. 305-310, 2016.

[7] Charvat, G.L., Fenn, A.J., and Perry, B.T., "The MIT IAP Radar Course: Build a Small Radar System Capable of Sensing Range, Doppler, and Synthetic Aperture (SAR) Imaging", IEEE Radar Conference, pp. 0138-0144, Atlanta, GA, 2012.

[8] Eid, A.M., "System Simulation of RF Front-End Transceiver for Frequency Modulated Continuous Wave Radar", International Journal of Computer Application, Volume 75, pp. 16-22, August, 2013.

[9] Gurbuz, S.Z., Ozcan, M.B., Panm, A.B., Demirhan, S., Hayran, Z., Karaduman, M.C., and Seyfioglu, M.S., "Target Detection and Ranging with the 2.4 GHz MTT Coffee Can Radar", IEEE $22^{\text {nd }}$ Conference on Signal Processing and Communications Applications, pp.1450-1453, 2014.

[10] Başarslan, O., and Yaldız. E., "Implementation of FMCW Radar for Training Applications", 4th International Conference on Electrical and Electronic Engineering, pp. 304-308, Ankara, 2017.

[11] Icoz, D., "Milimeterwave FMCW Radar Design", Master's Thesis, Middle East Technical University, December, 2009. 